\title{
Playful Interactive Systems to Support Physical Activity Among Wheelchair Users
}

\author{
Liam Mason \\ University of Lincoln \\ Brayford pool \\ Lincoln, LN6 7TS, UK \\ Lmason@lincoln.ac.uk
}

\begin{abstract}
Health benefits associated with physical activity (PA) are widely accepted; however, nearly twothirds of people with disabilities are not active enough to achieve health benefits. This is due to several unique environmental, programmatic and attitudinal barriers. Technology can be leveraged to reduce these barriers, e.g., Kinect being used to create movement-based games for children with mobility impairment. However, wheelchair-accessible games have not previously been built to address moderate-to-vigorous level exercise provision for wheelchair users. This PhD project will explore how to create game-based solutions to facilitate exercise and PA among wheelchair users, seeking to answer the following research questions: How do wheelchair users engage with PA and technology, how can we build playful systems to support wheelchair user's efforts to stay or become active, and can playful interactive systems facilitate positive exercise and PA experiences among wheelchair users? It is hoped that this work will actionable insights into the creation of game-based solutions to facilitate exercise and PA among wheelchair users.
\end{abstract}

Wheelchair, Physical activity, Thematic analysis, Integrated Behavioural Model, Playful technology.

\section{INTRODUCTION}

This $\mathrm{PhD}$ project seeks to explore technologies that can support physical activity (PA) among wheelchair users. Research has demonstrated the importance of regular PA (Kressler et al,.2014); however, nearly two-thirds of people with disabilities are not active enough to achieve health benefits. Benefits of PA are widely accepted; therefore, it is important to understand why some wheelchair users are not achieving recommended levels, encouraging them to become more active, along with supporting those who are already physically active, leveraging the potential of playful technology in this setting.

Previous literature encourages wheelchair users to engage in PA; however, they do not describe the initial challenges that wheelchair users experience (Ellapen et al., 2017). Reasons for this include unique environmental, programmatic and attitudinal barriers e.g. accessibility, financial and lack of knowledge (Stumbo et al., 2010). One solution to reduce barriers is with the use of technology e.g. pedometers, accelerometers, global positioning systems and heart rate monitors being used as motivational tools (Heyward and Gibson, 2014).

One popular method of facilitating PA with technology is through exergames, which Mueller et al. (2016) define as digital games that utilize physical exertion and effort as a key determinant in reaching the goal of the game (Mueller et al., 2017). Some examples of these exergames are Dance Dance Revolution (DDR), Wii Sports, Wii Fit and Kinect. Existing work in this field demonstrates that exergames can be a good form of physical exertion even when compared to traditional forms of exercise, with many fitness centers, schools, and senior centers are now offering interactive games to promote PA of children, adolescents, and older adults (Heyward and Gibson, 2014).

Exergames are well suited for playing alone or with others, requiring little training, providing an alternative to exercising in bad weather, and may serve as a transition to actually participating in sport and PA (Chamberlain and Gallagher 2008). However, commercially available exergames that are designed for wheelchair users are scarce (Rosly et al., 2017).

Finally, wheelchair-accessible games have not previously been built to address exercise provision for wheelchair-users implicating the need to explore how to create game-based solutions to facilitate exercise and PA among wheelchair-users.

Previous work has predominantly focused on the development of suitable interaction paradigms and the integration with game elements, but has not discussed the relationship of wheelchair users with 
PA and technology from a psychological perspective as such our approach builds on the Integrated Behavioural Model (IBM), this model will be further explained in a subsequent section, as a descriptive model that identifies the psychological factors that are highly likely to contribute to behaviour intention (De Angeli et al.,2016). Making it ideal for the elicitations of user requirements. In this work we will seek to answer the following questions:

- How do wheelchair users engage with PA and technology?

- How to build playful systems that can support wheelchair user's efforts to stay or become active?

- Can playful interactive systems facilitate exercise and PA among wheelchair users?

\section{RELATED LITERATURE}

Existing work on Barriers and facilitators for wheelchair users to engage in PA recognizes several elements, however, little work has involved technology. We also present an overview of literature that has involved wheelchair-accessible games.

\subsection{Barriers and facilitators for Wheelchair users to participate in PA}

Buffart et al. (2009) explored the main barriers and facilitators of PA in young adults with physical disabilities. They identified several barriers to engaging with PA including attitude, motivation, existing injury or fear of developing injuries, limited facilities, lack of information or knowledge. They also found that fun and socializing were facilitators of engaging in PA as well as improved health and fitness (Buffart et al., 2009).

Interestingly, the authors report some of some limitations that weaken the study. Firstly, that the results are based on a small sample size. Secondly that there may have been a bias in their participants as people that are more interested in PA and sports were more likely to participate in the study which may have resulted in the higher proportion of participating males compared with females. The fact that the authors chose to use focus groups also caused some limitations, most importantly the fact that they could not distinguish individual's data from a group.

Ellapen et al. (2017) reported a summary of 25 papers that indicated that sedentary wheelchair users have poor cardiometabolic risk profiles (because of a lack of PA, limiting their quality of life, characterised by low self-esteem, social isolation and depression (Ellapen et al., 2017). Wadey and Day (2017) also identified 10 themes in their work examining barriers, facilitators, and benefits to PA for amputees (Wadey and Day, 2017).

Ginis et al (2017) describe that wheelchair users may be unaware of, or unconvinced, of the painrelief benefits associated with an active lifestyle and may consider wheelchair sports inaccessible due to a perceived lack of wheelchair skills. Following on the authors state that previous research has found perceived behavioural control is not a significant direct predictor of PA in people with $\mathrm{SCl}$, which was consistent with their results, describing that the negative association might indicate that ambulators are overly confident in their sense of control over PA (Ginis et al., 2017).

\subsection{Technology used in playful interaction systems for wheelchair users}

The most prominent commercially available exertion game systems today are consoles such as Microsoft Xbox Kinect, Nintendo Wii, and Sony PlayStation Move. Research has found that they provide some physical health benefits (Rosly et al., 2017), however, they have been criticized for promoting the limited physical effort, especially when compared to existing sports activities. Mueller suggests one reason for this could be that the sensors or technology involved mainly aims to capture the movement of limbs as a generic input rather than focusing on specific exertion actions (Mueller et al., 2017).

This also leads on to results showing that the accessibility of motion-based games for persons using wheelchairs is limited, due to limitations of tracking and the lack of upper body gestures being used as input. Gerling et al. (2016) describe that recent efforts have been made to make camerabased systems more accessible by including the option to track seated players. However, this still limits the number of input options available and reduces the view of the player to the upper half of their body rather than recognizing the full person (Gerling et al., 2016).

There are many examples of research exergames involving wheelchair users in these, are predominately focusing on rehabilitation and fitness as opposed to designing for fun. Roslys (2017) work focused on using the PlayStation Move which compared exergame boxing to heavy bag boxing with the results showing that there was no significant physiological or perceived difference in responses between the two modalities of boxing (Rosly et al., 2017).

Gerling et al. (2013) developed KINECTWheels, a toolkit that facilitates the integration of wheelchairbased game input, demonstrating that wheelchairbased interaction is suitable for older and younger adults and that motion-based games can be designed in a way that makes wheelchair input 
both accessible and enjoyable (Gerling et al.,2013). Similarly, Eckert et al. (2017) developed Mokey which converts standard off the shelf software into exergames using a configurable set of gestures captured by a motion camera that is translated into keystrokes (Eckert et al., 2017).

Using KinectWheels, Hicks et al. (2015) created two exergames designed for children in wheelchairs. Both games are controlled by physically moving the wheelchair left or right to steer, whilst moving forward or backward to control the speed. The authors designed these games to be casual exergames stating that they provide easily accessible, intuitive gameplay that can be split up into short amounts of time, therefore making it easier to integrate gaming into daily routines. Concluding that participants were very enthusiastic and found it fun whilst being able to use the wheelchair inputs. However, not all participants enjoyed the genre of the game as participants stated a range of gaming preferences. The authors state this could be mitigated by providing an array of mini-games that span the more popular genres (Hicks et al., 2015).

Liberi is a cycling- based network game designed for children with cerebral palsy, it features several minigames that involve exertion. The data collected showed the games were fun, engaging and allowed the participants to reach recommended exertion levels (Ye et al., 2012). Likewise, GameWheels allows the ability to use the motion of the rollers to play video games on a personal computer allowing the use of commercial video games that are compatible with game joysticks or their variants (O'Connor et al., 2002). Unfortunately, no further work past 2006 can be found on this system.

Lastly, work has focused on the use of Fitbits with wheelchair athletes, describing that wearable fitness devices have demonstrated the capacity to improve PA. Participants showed an interest but there was a low adoption due to accessibility challenges (Carrington et al., 2015).

\subsection{Integrated Behavioural Model (IBM)}

It is important to choose a model that analyses psychological perspectives to understand underlying motives and design effective gamebased interventions. The intention to perform a specific behaviour has been considered by several theoretical models as a good indicator of the likelihood to engage in the behaviour. The Integrated Behavioural Model (IBM) is the combination of two theories; the theory of planned behaviour (TPB) and the theory of reasoned action (TRA). The IBM states that there are five components that directly affect a person's behaviour. The first and most important determinant is the intention, without intention to do so, an individual is unlikely to carry out a behaviour. Behavioural intention is determined by attitude, perceived norms, and personal agency. However, unlike the TRA and TPB, the IBM also suggests that other factors such as knowledge, environmental constraints, habit, and salience influence the likelihood to engage in the behaviour (Motaño and Kasprzyk, 2008).

The IBM has been extensively validated in empirical research, for example, it has been used in healthcare to promote HIV prevention, understand binge drinking and to facilitate PA among children. Interestingly, even though the IBM has previously been applied to PA and tested amongst several demographics it has not currently been tested with a sample of wheelchair users; thus, our study contributes to the growing literature of theory-based determinants of PA amongst wheelchair users.

De Angeli (2016) also demonstrated how the IBM can be used as a method of gathering user requirements, stating that it provided rich information on motivations and barriers of older people towards activities, concluding that the IBM was efficient, reliable and easy to link to design thinking (De Angeli et al.,2016). This work will also seek to leverage the IBM as a theoretical framework for further exploration of perspectives of wheelchair users on PA and the potential of technology in this setting.

\section{PROBLEM STATEMENT AND GOALS OF THE PHD RESEARCH}

From existing work, we formulate several problems with the current state of research involving the development of playful interactive technology to support PA among wheelchair users.

- Wheelchair-accessible games have not previously been built to address moderateto-vigorous levels of exercise for wheelchair users, but focus on game mechanics and physical stimulation instead

- Lack of in-the-wild evaluation of long-term system uptake for existing solutions.

- The absence of technology that supports outdoor interaction for wheelchair users.

We will seek to address these issues through these following steps that will be explained in more detail in subsequent sections:

- Firstly, to explore how wheelchairs users engage with PA and technology using interviews and an online survey, from which we will derive design recommendations for the development of activity-supporting systems for people with mobility impairments.

- Using these design requirements, the PhD project will create a playful system that 
supports exercise among wheelchair users for indoor home use.

- Similarly, as the previous step, the PhD project will create a playful system that supports exercise among wheelchair users suited for outdoor use.

Exertion games are a relatively young field; hence most studies are conducted in laboratories, if studies were conducted in "the wild", they were often short exposures (Rogers, 2011). Mueller et al. (2016) justify this, stating that studies often involve fragile or expensive technology that works best under controlled conditions. This is especially prevalent with work that involves wheelchair users with only a few examples conducted out of a lab environment.

There is also a lack of exertion games which support outdoor interaction. The points Mueller made were directed towards exergames as a whole which is mainly designed with able-bodied users in mind (Mueller et al., 2016).

The main goal of our work is to explore how wheelchair users currently engage with PA, and how to build playful systems that can support their efforts to stay or become active. The work will be split into three studies, firstly we will seek to define user requirements, which will then be used to design and develop two playful interactive systems that can be used in a non-lab setting i.e. a user's home or a park.

\section{WORK IN PROGRESS}

This section describes the initial studies methodology and current results.

\subsection{Step 1: Understanding how playful systems can support PA (Physical Activity) among wheelchair users.}

The first study of the $\mathrm{PhD}$ explores perspectives of wheelchair users on PA and the potential of technology in this setting, seeking to address the research questions:

How do wheelchair users engage with PA and technology, and How to build playful systems that can support their efforts to stay or become active?

We present results from interviews in which wheelchair users were asked to discuss PA and technology.

\subsection{Method}

Using the IBM, as it provides rich information on motivations and barriers, we conducted semistructured interviews with wheelchair users to elicit the specific components of the IBM. This was used to identify and explore barriers and facilitators associated with PA and technology.
We will then create an online survey to reach a larger demographic to validate the interview outcomes and make sure our findings our generalizable.

\subsection{Participants}

The interviews were conducted with a total of 8 participants ( 2 females, 6 males; age ranging from 20 to 61 years old, mean 33.8; 4 lived in rural areas, 3 from urban and 1 that moved between rural and urban life; 6 lived in the UK, 1 lived in Latvia and 1 lived in Norway; all participants lived with someone either family, partners or flatmates; all were manual wheelchair users with a range of disabilities; wheelchair use ranged from 2 to 35 years, mean 11 years. Ethical approval was granted through the University of Lincolns ethical board.

\subsection{Procedure}

To outline and validate the interviews, a pilot study was conducted with three non- wheelchair users (due to population size). This lead to some questions being reworded, refined and in some cases removed, additional probes were also put in place were necessary to allow for better flow. Participants were recruited locally through local clubs and from Social media via Twitter using accessibility hashtags. The interviews were conducted either in person at a suitable location chosen by the participant or via telecommunications applications of the participants choosing.

At the start of the interview, participants were presented with an overview of the study and given a consent form to sign. The interviews were split into three sections; General, IBM, and technology. The general questions sough to gain insight into the participant's life asking demographical questions as well as questions relating to PA to help with probes in later sections. The IBM questions elicited questions based on the model. The last section of the interviews sought to gain knowledge of how participants interacted with technology.

\subsection{Analysis}

Data were analysed using Thematic analysis following both a deductive and inductive approach as laid out by Braun and Clarke (Braun and Clarke 2006). Firstly, all the statements relevant to IBM's constructs that describe behavioural intention were extrapolated in a deductive manner. Codes were constructed according to the IBM and gathered into 5 code-families: all were labeled as the original categories of the theoretical model (Activity, Attitude, Perceived norm, and Personal Agency). Each code family was further enriched with specific codes. An inductive approach was used to discover themes related to barriers of using technology. 


\subsection{Results}

Participants reported a high amount of salience and positivity when describing the benefits of PA, elaborating on physical and mental wellbeing. Participants mentioned several social aspects which affect PA such as others motivating or demotivating them. Defying the stereo type was mentioned often with the word "Spite" being used to describe the disregard of others negative thoughts, participants also negatively stated how they could not compete in some cases with able-bodied users which resulted in not participating. Participants also demonstrated a sense of empathy with other wheelchair users.

Participants reported a high amount of control when performing in PA with some demonstrating a high knowledge not only their own skill but others as well taking on a coaching position. However, most participants mentioned in some way how having a disability makes PA harder either daily or as an entry level barrier. Specialized equipment and exercises were mentioned frequently which resulted in mixed responses with some participant's liking their specialized chair and others experiencing pain and discomfort when performing specialized exercises. Cost time and Environmental constraints such as weather and accessibility were reported as significant barriers to performing in PA.

Routine was also strongly evident throughout with all participant's mentioning some routine they had with PA, interestingly there was little evidence of spontaneous exercise. Five themes related specifically to barriers to technology where discovered; Stigma towards gaming, Brand loyalty, user behaviour, technology behaviour and Theme.

\subsection{Discussion}

As discussed in the related work several studies have been done analysing barriers to PA for wheelchair users with many finding themes that are similar to the results found in this study. However, the video game and technology related themes found are to the best of our knowledge are unique, suggesting additional barriers such as stigma, brand loyalty, user/ technology behaviour and theme exist and are important to consider when designing system.

To validate our interview findings outcomes an online survey will be created using the results gathered to address the limitations of the sample size and demographic. This will reflect on the IBM in the context of wheelchair and technology use to derive design requirements for the development of activity-supporting systems for people with mobility impairments. Contributes to the growing literature of theory-based determinants of PA amongst wheelchair users. This provides an empirically grounded set of adult wheelchair user requirements. Which in turn will answer our research questions; How do wheelchair users engage with PA and technology? And How to build playful systems that can support wheelchair user's efforts to stay or become active? Once complete, we plan to submit this work to $\mathrm{CHI} 2019$.

\section{FUTURE WORK}

This section will outline the future work of the PhD, which includes a brief summary of implementation steps and studies.

\subsection{Developing an playful interactive system to facilitate PA for a wheelchair for indoor use}

This part of my PhD research will seek to create an interactive system based on the results of the initial study that uses non-portable movement-tracking technology that can be deployed in a user's home. Facilitating user input is important so it is vital the appropriate technology is chosen, potential options are; Gesture based systems e.g. the Kinect and MYO connect, Controller based e.g. PS Move, Wii, Switch (Docked) and VR systems (HTC VIVE and Oculus Rift) and there is also the possibility of creating a custom Arduino system. The development environment will be dependent on the chosen technology i.e. Most can be developed in Unity3D with $\mathrm{C \#}$ except for the Switch which has a private Devkit, so created systems would be created with inbuilt tools e.g. Nintendo Labo or FUZE4.

We expect several challenges related to this work such as: designing technology suited for home use as initial results showed some participants didn't have room to use some devices. As a long-term study in the user's home is planned it is further paramount that the game is of a finished standard (offering tutorials and help in the researcher's absence), suggesting the need to implement a vertical slice. Likewise, replayability will play an important role as initial results and previous literature showed game themes influenced participants. As a mitigation strategy, we plan to implement several mini-games in line with previous research (e.g., Hicks et al., 2015). Additionally, results from the previous study showed technology behaviour (not working as intended) and Player behaviour (cheating) are themes that should be designed for. Lastly, as we are looking to recruit 56 participants for this step of the research it is also important that the required amount of systems is available for use for a long-term study, possibly being a deciding factor when choosing a technology for implementation.

To begin with, short-term studies will be conducted to evaluate the effectiveness of each deliverable. Afterward, we will seek to recruit a small number of participants allowing them to play the created system in their own home as part of a long-term 
study. To evaluate the results with a focus on longterm engagement, overall enjoyment and perceived exertion, we will use semi-structured interviews, game metrics and standardized questionnaires such as the BORG (Borg, 1998) rating of perceived exertion and PENS (Player experience of needs satisfaction) questionnaire (Ryan and Rigby.,2006).

The expected outcomes of this work are hoped to contribute to the question whether playful interactive systems can facilitate exercise and PA. This part of the research will be carried out during Q4 2018 and Q1 2019 as seasons do not affect the feasibility of the study.

\subsection{Developing an playful interactive system to facilitate PA for wheelchair users for outdoor use}

Building on the first two steps of this PhD project, it is planned to create an interactive system that uses portable movement tracking technology to facilitate use in an outdoor setting. We can expect to create a location-based, augmented reality system; we will integrate portable technology such as smart phones, tablets, Nintendo Switch, Fitbit, Smartwatches or an Arduino system. Likewise, to the indoor technology the development kit will vary depending on chosen technology.

This shares some challenges as the indoor study, however, it is important to note that unlike a user's home, outdoor use adds several additional barriers both to technology and users i.e. social barriers and technological requirements. So vigorous testing is needed because it is important it works as intended.

Input is also important as initial results showed users had problems using technology whilst on the move i.e. games like Pokémon go that have mechanics requiring users to move whilst looking at their device, this is not always possible for wheelchair users. As no planned to be a long-term study there is less of a need for a finished system, so a vertical slice will be used to save time on development.

A short-term study will first be done again to examine the effectiveness of the deliverable. We will seek to recruit $8-12$ participants and ask them to play the created system. This study will seek to answer if the created system can provide a suitable amount of exertion to meet recommended exercise requirements for wheelchair users whilst being fun and reusable. Participants enjoyment and exertion levels will be recorded through semi-structured interviews, game metrics and standardized questionnaires such as the PENS and BORG.

The expected outcomes of this work are again, hoped to contribute to the question whether playful interactive systems can facilitate exercise and PA. This part of the research will be carried out during Q2 and Q3 2019 due to summer making outdoor based systems for feasible.

\subsection{Ideation Process}

It is important that the games created are of good quality as well as also being fun and repayable, to achieve this multiple academic industry inspired methodologies will be used. Firstly, Guardiola's (2016) Gameplay Loop which breaks down a game into loops making it applicable not only for design purposes but also for analysis allowing for a breakdown of exergame titles, such as Pokémon's Go's Main gameplay loop of Explore, encounter, capture, collect, level up and upgrade Pokémon (Guardiola, 2016).

Secondly, Librande's (2010) one-page design document will assist in creating designs that are easily communicated within a team which compliments the visual side of the gameplay loop allowing for easy integration (Librande, 2010).

It is also important to note that creating games for wheelchair users offers additional challenges, so some form of participatory design will be incorporated as literature has shown that the involvement of design experts leads to more refined concepts, and the involvement of end users provides valuable insights into their preferences and needs. Once suitable designs are chosen the games will be created using suitable software i.e. Unity3D.

\section{HOPES FOR THE DC}

Attending the British $\mathrm{HCl} 2018$ doctoral consortium would provide me with a range of opportunities allowing me to grow as a researcher. Firstly, it would be an opportunity to present and share my work with researchers from other institutions, offering insights beyond the research philosophy I am currently familiar with, and providing me with opportunities to receive feedback on yet to be defined elements of my work (e.g., choice of technology in different settings). Furthermore, the DC would strengthen my presentation skills in an academic setting, hopefully providing new perspectives and creative ideas.

I also enjoy learning about what others are doing, especially in the $\mathrm{HCl}$ field so this will give me an opportunity to learn and connect with other students in a similar field.

Finally, I currently expect to finish my PhD by the end of 2020, so attending the consortium will give me the opportunity of networking and exploring future career paths (e.g., understanding how to prepare for an academic career throughout the $\mathrm{PhD}$ ), allowing me to set further goals.

\section{REFERENCES}

Borg, G., 1998. Borg's perceived exertion and pain scales. Human Kinetics. 
Buffart, L.M., Westendorp, T., Van Den BergEmons, R.J., Stam, H.J. and Roebroeck, M.E., 2009. Perceived barriers to and facilitators of physical activity in young adults with childhoodonset physical disabilities. Journal of Rehabilitation Medicine, 41(11), pp.881-885.

Carrington, P., Chang, K., Mentis, H. and Hurst, A., 2015, October. But, I don't take steps: Examining the Inaccessibility of Fitness Trackers for Wheelchair Athletes. In Proceedings of the 17th International ACM SIGACCESS Conference on Computers \& Accessibility (pp. 193-201). ACM.

Chamberlin, B. and Gallagher, R., 2008. Using Video Games to Promote Physical Activity.

De Angeli, A., Cozza, M., Jovanovic, M., Tonolli, L., Mushiba, M., McNeill, A., Coventry, L., Garschall, I.M., Hamm, T., Hornung, D. and Müller, C., 2016. Understanding Motivations in Designing for Older Adults. In 12th COOP-International Conference on the Design of Cooperative Systems.

Eckert, M., Gómez-Martinho, I., Meneses, J. and Martínez, J. (2017). New Approaches to Exciting Exergame-Experiences for People with Motor Function Impairments. Sensors, 17(2), p.354.

Eckert, M., López, M., Lázaro, C. and Meneses, J., 2017. MoKey: a versatile exergame creator for everyday usage. Assistive Technology, pp.1-10.

Ellapen, T.J., Hammill, H.V., Swanepoel, M. and Strydom, G.L., 2017. The health benefits and constraints of exercise therapy for wheelchair users: A clinical commentary. African journal of disability, 6(1), pp.1-8.

Gerling, K.M., Kalyn, M.R. and Mandryk, R.L., 2013, April. KINECT wheels: wheelchair-accessible motion-based game interaction. In $\mathrm{CHI} 13$ Extended Abstracts on Human Factors in Computing Systems (pp. 3055-3058). ACM.

Gerling, K.M., Mandryk, R.L., Miller, M., Kalyn, M.R., Birk, M. and Smeddinck, J.D., 2015. Designing wheelchair-based movement games. ACM Transactions on Accessible Computing (TACCESS), 6(2), p.6.

Guardiola, E., 2016, November. The Gameplay Loop: a Player Activity Model for Game Design and Analysis. In Proceedings of the 13th International Conference on Advances in Computer Entertainment Technology (p. 23). ACM.

Heyward, V.H. and Gibson, A., 2014. Advanced fitness assessment and exercise prescription 7th edition. Human kinetics.

Hicks, K. and Gerling, K., 2015, October. Exploring casual exergames with kids using wheelchairs. In Proceedings of the 2015 Annual Symposium on Computer-Human Interaction in Play (pp. 541-546). ACM.
Kressler, J., Cowan, R.E., Bigford, G.E. and Nash, M.S., 2014. Reducing cardiometabolic disease in spinal cord injury. Physical Medicine and Rehabilitation Clinics, 25(3), pp.573-604.

Librande, S. (2010). One-Page Designs.

Martin Ginis, K.A., Papathomas, A., Perrier, M.J., Smith, B. and Shape-Sci Research Group, 2017. Psychosocial factors associated with physical activity in ambulatory and manual wheelchair users with spinal cord injury: a mixed-methods study. Disability and rehabilitation, 39(2), pp.187192.

Montano, D.E. and Kasprzyk, D. (2008) Theory of reasoned action, theory of planned behavior, and the integrated behavioral model. In: Glanz, K., Rimer, B.K. and Viswanath, K. Eds., Health Behavior and Health Education, Jossey-Bass, San Francisco, 67-96.

Mueller, F., Khot, R.A., Gerling, K. and Mandryk, R., 2016. Exertion games. Foundations and Trends ${ }^{\circledR}$ in Human-Computer Interaction, 10(1), pp.1-86.

O'Connor, T.J., Fitzgerald, S.G., Cooper, R.A., Thorman, T.A. and Boninger, M.L., 2002. Kinetic and physiological analysis of the GAME (Wheels) system. Journal of rehabilitation research and development, 39(6), p.627.

Rogers, Y., 2011. Interaction design gone wild: striving for wild theory. Interactions, 18(4), pp.5862.

Rosly, M., Halaki, M., Rosly, H., Cuesta, V., Hansan, N., Davis, G. and Hasain, R. (2017). Commercially available exergames that are for wheelchair-bound individuals with spinal cord injury (SCl) are scarce. games for health.

Rosly, M., Rosly, M., N, H., GM, D. and R, H. (2017). Exergaming boxing versus heavy-bag boxing: are these equipotent for individuals with spinal cord injury?.

Ryan, R.M., Rigby, C.S. and Przybylski, A., 2006. The motivational pull of video games: $A$ selfdetermination theory approach. Motivation and emotion, 30(4), pp.344-360.

Stumbo, N.J., Hedrick, B.N., Weisman, C. and Martin, J.K., 2010. An exploration into the barriers and facilitators experienced by university graduates with disabilities requiring personal assistance services. Journal of Science Education for Students with Disabilities, 14(1), p.2.

Wadey, R. and Day, M., 2017. A longitudinal examination of leisure time physical activity following amputation in England. Psychology of Sport and Exercise. 
Ye, Z., Hernandez, H.A., Graham, T.C., Fehlings, D., Switzer, L., Hamza, M.A. and Schumann, I., 2012, October. Liberi and the racer bike: exergaming technology for children with cerebral palsy. In Proceedings of the 14th international ACM SIGACCESS conference on Computers and accessibility (pp. 225-226). ACM. 